\title{
Enabling Highly Stretchable Polymer Semiconductor Films through Nanoconfinement Effect
}

Jie $\mathrm{Xu}^{1, \dagger}$, Sihong Wang ${ }^{1, \dagger}$, Ging-Ji Nathan Wang ${ }^{1}$, Chenxin $\mathrm{Zhu}^{2}$, Lihua $\mathrm{Jin}^{3,4}$, Xiaodan $\mathrm{Gu}^{1}$, Shucheng Chen ${ }^{1}$, John W.F. To ${ }^{1}$, Simon Rondeau-Gagné ${ }^{1}$, Bob C. Schroeder ${ }^{1}$, Chien $\mathrm{Lu}^{1}$, Yanming Wang ${ }^{5}$, Wei Cai ${ }^{3}$, Christian Linder ${ }^{4}$, Yun-Hi Kim ${ }^{6}$, He Yan ${ }^{7}$, Gi Xue ${ }^{8}$, Jeffery B.-H. Tok ${ }^{1}$, Jong Won Chung ${ }^{1,9, *}$, Zhenan Bao ${ }^{1, *}$

'Department of Chemical Engineering, Stanford University, Stanford, CA 94305, USA ${ }^{2}$ Department of Electrical Engineering, Stanford University, Stanford, CA 94305, USA

${ }^{3}$ Department of Mechanical Engineering, Stanford University, Stanford, CA 94305, USA

${ }^{4}$ Department of Civil and Environmental Engineering, Stanford University, Stanford, CA 94305, USA

${ }^{5}$ Department of Materials Science and Engineering, Stanford University, Stanford, CA 94305, USA

${ }^{6}$ Department of Chemistry and RINS, Gyeongsang National University, Jinju, 660-701, South Korea

${ }^{7}$ Department of Chemistry, The Hong Kong University of Science and Technology, Clear Water Bay, Kowloon, Hong Kong

${ }^{8}$ Department of Polymer Science and Engineering, Institute of Chemistry and Chemical Engineering, Nanjing University, Nanjing 210093, China,

${ }^{9}$ Samsung Advanced Institute of Technology Yeongtong-gu, Suwon-si, Gyeonggi-do 443-803, South Korea

*Corresponding Author, Email: zbao@stanford.edu (Z.B.); jongwon@stanford.edu (J.W.C.)

$\dagger$ These authors contributed equally to this work.

The lack of stretchable semiconductors has limited the development of stretchable and wearable electronics. All the existing approaches typically sacrifices charge-transport mobility. Here, we present a concept based on nanoconfinement effect of polymers to significantly improve the stretchability of polymer semiconductors, without affecting its charge transport mobility. The increased polymer chain dynamics under nanoconfinement significantly reduces the plastic modulus of the conjugated polymer and largely defers the onset crack formation under strain. As a result, our fabricated semiconducting film can be stretched up to $100 \%$ strain without affecting its mobility, through which a 
record-high mobility of $1.32 \mathrm{~cm}^{2} / \mathrm{Vs}$ has been achieved at $100 \%$ strain. The fully stretchable transistors also exhibit very high bi-axial stretchability, which has been further demonstrated as a skin-like finger-wearable driver for a LED. Because of high versatility on different semiconducting polymers, our nanoconfinement concept could be utilized to impart high stretchability onto any molecular-engineered high-performance conjugated polymers that are developed in the future.

Electronics for biomedical applications, such as physiological monitoring (1), implanted treatment (2), electronic skins (3) and human-machine interface (4), need to be mechanically compatible with biological tissues, with characteristics of low modulus, flexibility, and stretchability. Several approaches based on geometric designs, such as buckles $(5)$, microcracks $(6,7)$ and kiragami $(8)$, has been demonstrated to impart stretchability to electronics for a variety of wearable applications. In addition, stretchable electronics based on intrinsically stretchable materials will enable scalable fabrication, higher device density, and better strain tolerance. However, stretchable electronics remain scarce due to the lack of high-performance stretchable semiconducting materials that possess both high mechanical ductility and high carrier mobility at large strains. In recent years, conjugated polymers have been demonstrated with high charge carrier mobilities rivaling that of poly-Si.(9-11) However, they have poor stretchability. Molecular design rules (12) (13) that are effective in improving stretchability often results in decrease in mobility. Nanowire/nanofibril network $(14,15)$ and micro-cracked films (6) have been employed to maintain charge transport under stretching, but often 
conferring limited strain tolerance improvements. Thus, fundamentally different strategies bearing new scientific concepts are highly desirable for a marked advancement in this field.

It has been known that nanoconfinement can alter many physical properties of polymers, including decrease in mechanical modulus $(16,17)$, glass transition temperature $(18,19)$, and increase in mechanical ductility $(20-22)$. These changes are primarily due to the enhanced polymer chain dynamics in the amorphous regions (23-25) and the restricted growth of large crystallites (26). Therefore, we hypothesized that the nanoconfinement effect may increase the mechanical stretchability of polymer semiconductors.

Here, we report the application of the nanoconfinement effect to develop highly stretchable polymer semiconductors possessing record-high performances at high strains. We realized this via a phase separation process between the conjugated polymer and a compatible elastomer (termed CONPHINE), to result in desired nanoscale semiconducting polymer network with low crystallinity to reduce modulus and fiber-like large aggregates to allow good charge transport (Fig. 1A), which also possesses a large area of deformable interfaces. Such a design provides significant improvement to the stretchability of existing high-mobility polymer semiconductors. Even when under $100 \%$ strain, our generated CONPHINE semiconducting film exhibited no cracks and negligible decrease in charge carrier mobility, while maintaining a record-high mobility of $1.32 \mathrm{~cm}^{2} / \mathrm{Vs}$ at $100 \%$ strain, a value that is one order of magnitude higher than the current best reported for any stretchable organic 
semiconductors in literatures $(6,14,15,27-29)$. Compared to the corresponding neat film, the CONPHINE film showed a 3-orders of magnitude improvement in mobility under $100 \%$ strain. Furthermore, fully stretchable transistors with CONPHINE semiconducting layer offered a record-high mobility of $0.55 \mathrm{~cm}^{2} /$ Vs even at $100 \%$ strain. Ion-gel gated organic transistors have higher mobilities. (30) However, they have limitations in switching speed and stability issues due to electrochemical reactions. With our high-performance stretchable transistors, we proceed to demonstrate a finger-wearable driver circuit for a light-emitting diode (LED). Importantly, we also show that our described nanoconfinement-based approach is generally applicable to several high-mobility conjugated semiconducting polymers, which brings the mobilities of four resulting films over $1 \mathrm{~cm}^{2} / \mathrm{Vs}$ at $100 \%$ strain. Taken together, this work represents a promising and general physical engineering approach to fabricate high performance stretchable semiconductors.

We first investigate poly[3,6-di-2-thienyl-pyrrolo[3,4-c]pyrrole-1,4-dione-alt-thieno[3,2-b]thiophene] (DPPT-TT; 1) (31) as the high-mobility semiconducting polymer and polystyrene-block-poly(ethylene-co-butylene)-block-polystyrene (SEBS) as the soft elastomer (Fig. 1B), due to their comparable surface energies to ensure a nanoscale mixed morphology (Fig. S1, Table S1). The improvement in mechanical ductility using the nanoconfinement effect together with the deformable interfaces is first investigated on three film models of 1: (i) thick neat film $(\sim 135 \mathrm{~nm})$, (ii) thin neat film $(\sim 35 \mathrm{~nm})$ with only the nanoconfinement effect, and (iii) thin film/SEBS bilayer 
film with both the nanoconfinement effect and a deformable interface (Fig. 1C). A significant decrease in the elastic modulus, and a significant increase in the onset strains of plasticity and cracks were observed from thick to thin film (Figs. 1D and S2-6). The addition of a deformable SEBS interface further decreased the elastic modulus and increases the onset strains, from the thin film to the bilayer film. A suppressed crystallization was observed by grazing incidence X-ray diffraction as the films are from thick to thin and finally to thin/SEBS bilayer (Fig. S3). This should also contribute to the reduction of the modulus of the corresponding films. Furthermore, the effective modulus of the DPPT-TT nanofiber is calculated to be only around $190 \mathrm{MPa}$, which is 3 times lower than the bilayer film and almost ten times lower than the thick film because of a stronger 2-dimensional nanoconfinement effect (Fig. 1D and Fig. S4). On the molecular level, the dichroic ratios from UV/Vis spectroscopy for the thin and the bilayer films all exhibited an increase of the chain alignment with increasing applied strains. On the other hand, the thick film showed little dichroic ratio since strain is relieved via crack propagations (Figs. 1E and S7). The improved stretchability can be directly observed from the significantly lowered crack width in the thin film and the bilayer film, as compared to the thick film (Fig. S8). Compared to the thin film model, the 2-dimensional nanoconfinement effect in the DPPT-TT nanofiber film should further improve the mechanical ductility, and thus give an even smaller crack size at 100\% strain (Fig. S8). Hence, our observations validated the benefit from the nanoconfinement effect, together with a compatible elastomer, can significantly improve the stretchability of polymer semiconductors. 
A

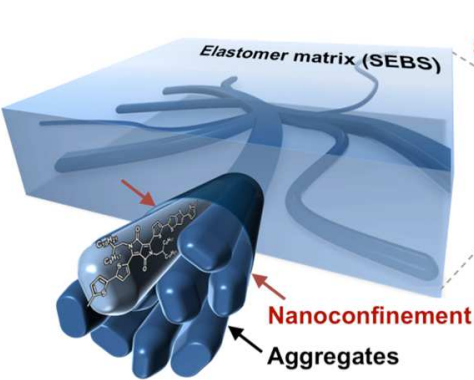

C

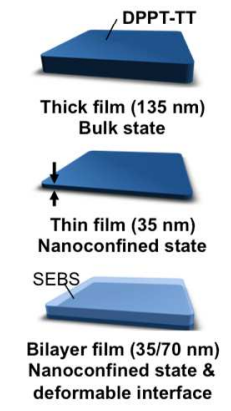

$\mathbf{F}$
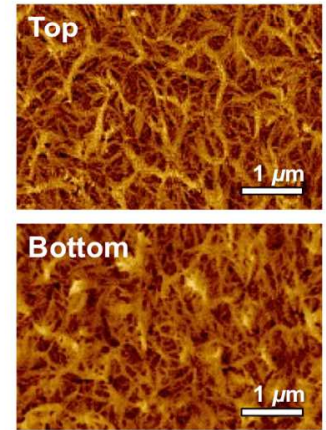

Stretchable

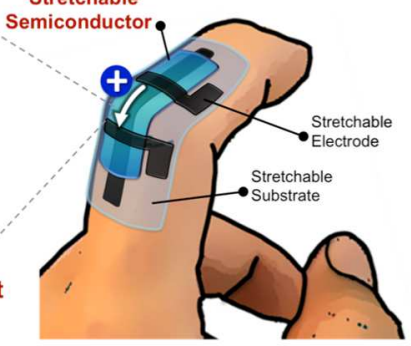

B

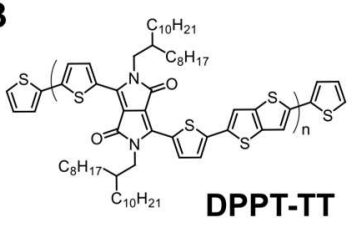

(1)

SEBS

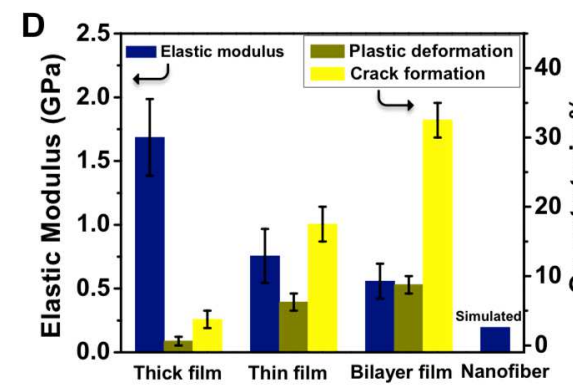

E

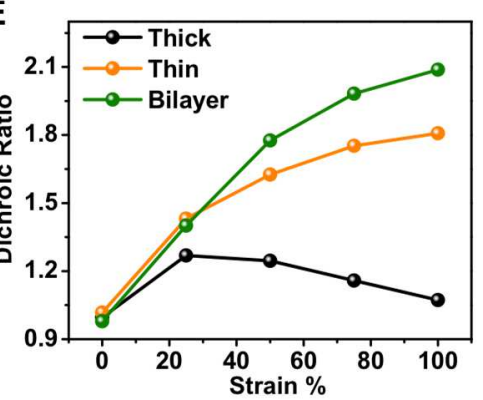

H
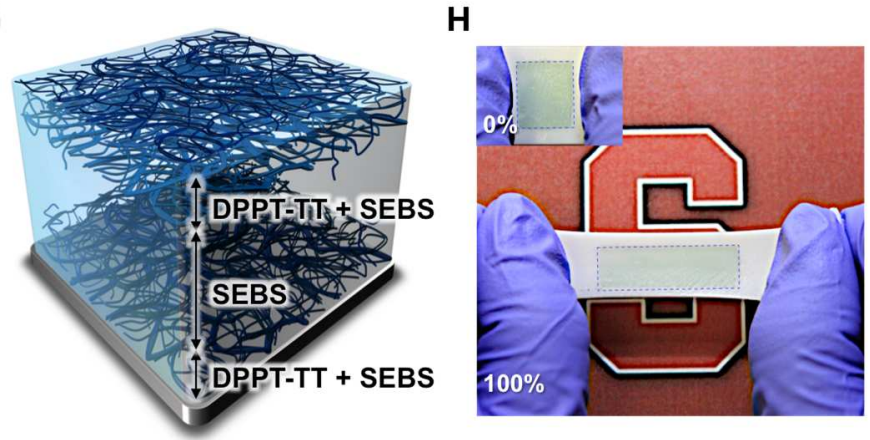

Fig. 1. Nanoconfinement effect is used to enhance the stretchability of polymer semiconducting film through CONPHINE method. (A) 3D schematic of the desired morphology comprised of embedded nanoscale networks of polymer semiconductor to achieve high stretchability, which can be used to construct highly stretchable and wearable thin-film transistor. (B) Chemical structures of semiconducting polymer DPPT-TT (labeled as 1) and SEBS elastomer. (C) Schematic diagram showing three types of semiconducting films for investigation of the role of nanoconfinement effect. (D) Elastic moduli, onset strain of plasticity, and onset strain of crack of films from (C). (E) Dichroic ratios (R) as a function of strain for films from (C). (F) AFM phase images of the top and bottom interfaces of the CONPHINE-1 film with $70 \mathrm{wt} \%$ SEBS. (G) 3-D illustration of the morphology of the CONPHINE-1 film. (H) Photographs of a CONPHINE-1 film (the blue area) at $0 \%$ strain and stretched to $100 \%$ strain on a rubber substrate. 
To obtain the desired nanoconfinement morphology (Fig. 1A) with deformable interfaces, possible fabrication techniques include photolithographic-patterning (32), nanostructure-templating (33) and phase separation (34). Among them, only the phase separation method can simultaneously introduce all desirable features in one step $(6$, 35) and is more scalable. Here, we describe a novel conjugated-polymer-elastomer phase-separation induced engineering (CONPHINE) to achieve the desired morphology.

As observed by the atomic force microscopy (AFM) phase images of the CONPHINE-1 film, the desired fiber network is formed with smaller nanofiber diameters at higher SEBS concentrations (Fig. S9). At $70 \mathrm{wt} \%$ of SEBS, the nanofiber diameter become small enough $(<50 \mathrm{~nm})$ to give a strong nanoconfinement effect at both the top and bottom surfaces (Fig. 1F). Unlike the pre-grown nanowires/nanofibers, these phase-separation formed nanofibers have reduced crystallinity but high aggregation (Fig. S10), which ensures both enhanced stretchability and good charge transport. As revealed by X-ray photoelectron spectroscopy (XPS, Fig. S11), the layer between these two interfaces is primarily occupied by SEBS. This tri-layer morphology is depicted in Fig. 1G. As shown in Fig. $1 \mathrm{H}$, this CONPHINE-1 film can be stretched up to $100 \%$ strain on a rubbery substrate without any visible cracks.

The stretchability of the CONPHINE-1 film is studied by first applying a $100 \%$ strain on a polydimethylsiloxane (PDMS) supported film (Fig. 2A), followed by 
observation under optical microscope after being transferred to a Si substrate. As shown in Fig. 2B, the CONPHINE-1 film can be effectively stretched to $100 \%$ without resulting in any crack formations. Despite of inhomogeneous deformations that result in thickness variation as evidenced in Fig. S12, the overall film's continuity was not affected as observed by the AFM image at a higher magnification (inset image of Fig. 2A). In comparison, the neat-1 film at 100\% strain developed big cracks with widths around $20 \mu \mathrm{m}$.

The electrical performance of the CONPHINE-1 film functioning as a stretchable semiconductor was subsequently measured in a thin-film transistor (TFT) by soft contact lamination (27) on a bottom-gate-bottom-contact stack: OTS-assembled $\mathrm{SiO}_{2}$ (gate dielectric) coated highly-doped $\mathrm{Si}$ (gate), with Au contacts on the top (Figs. 2C \& S13). By comparing the mobility from the CONPHINE-1 film with different SEBS percentages, it was determined that $70 \mathrm{wt} \%$ SEBS provides the optimum condition to enable the highest mobility at $100 \%$ strain (Figs. S14). The stretchability of this CONPHINE-1 film was then systematically studied under different strains. As shown in Fig. 2D(i), the CONPHINE-1 film (i.e. $70 \mathrm{wt} \%$ SEBS) gives similar transfer property as the neat-1 film (i.e. 0\% SEBS), which clearly demonstrates that this approach, unlike the other existing approaches for improving the stretchability, doesn't sacrifice the semiconductor's electrical performance. When both films are stretched to $100 \%$ strain parallel to charge transport, the transfer curve of the CONPHINE film remains unaffected, with its on-current $\sim 3$ orders higher than the severely degraded value from the neat-1 film (Figs. 2D(ii) \& S15). In addition, the 
CONPHINE-1 film showed no decrease in the mobility during the entire stretching process (Fig. 2E), in which its mobility at $100 \%$ strain reached a record-high value of $1.32 \mathrm{~cm}^{2} / \mathrm{Vs}$ in maximum, and $1.08 \mathrm{~cm}^{2} / \mathrm{Vs}$ on average, which is $\sim 3$-order improvement compared to the neat-1 film. This significant improvement in its stretchability stems from the well-maintained semiconducting nanofiber network upon stretching, as revealed by the AFM images (Fig. S16). On the other hand, when the stretching is perpendicular to the charge transport direction, we observed that there is only a very small decrease in both the on-current and the mobility from the CONPHINE-1 film at 100\% strain (Figs. 2D(iii) \& 2F). Again, this observation is a significant improvement compared to the $\sim 2$ orders decrease in the neat film. To further probe the film's stretchability, we observed that the CONPHINE-1 film can be even stretched to $200 \%$ strain, with its mobility maintained at $0.33 \mathrm{~cm}^{2} /$ Vs (Fig. S17). Compared to previously reported mobilities obtained under stretching in literature (summarized in Fig. 2G), our CONPHINE-1 film affords not only a mobility approximately one order higher at $100 \%$ strain, but also a maintained performance at even much larger strains. 

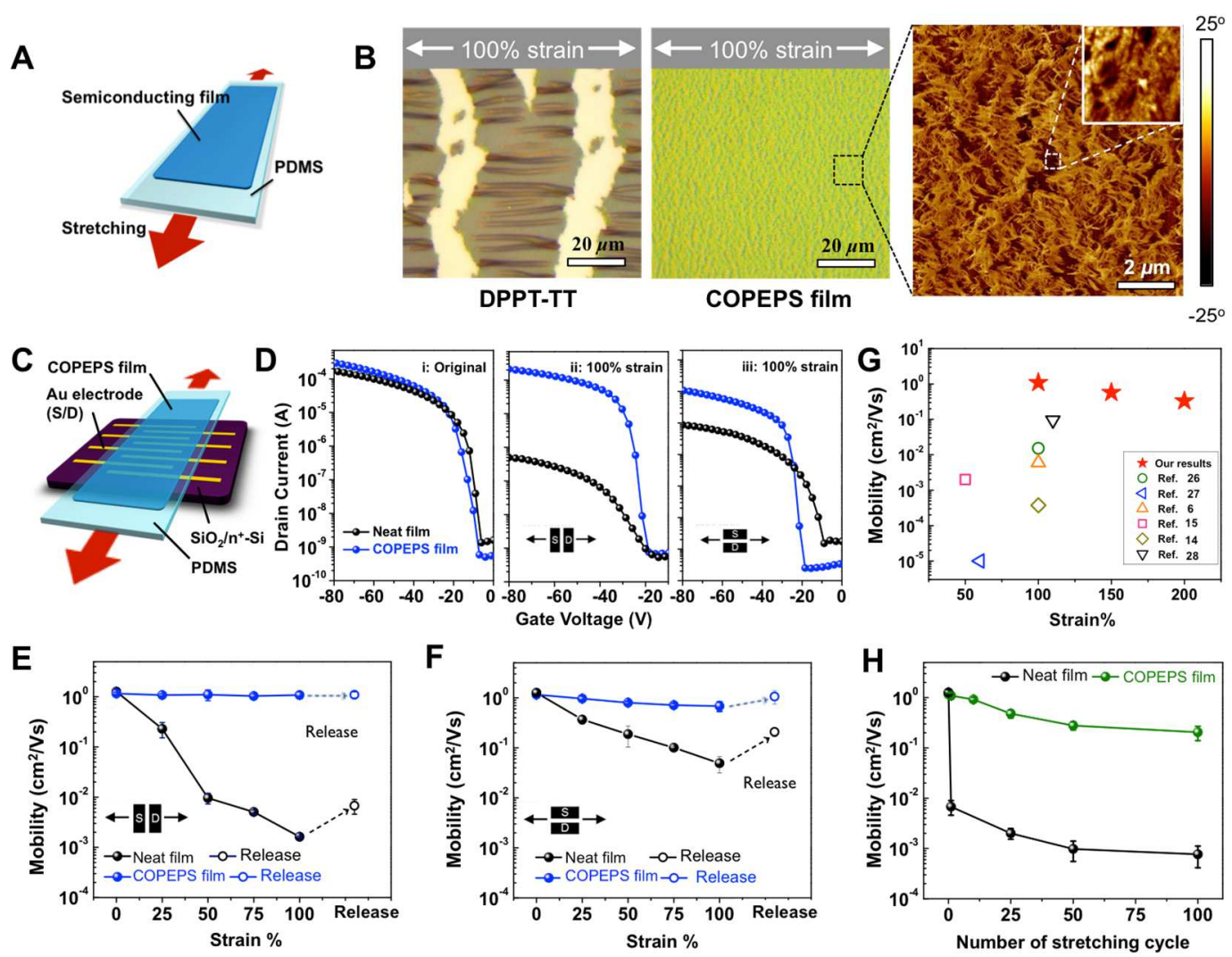

Fig. 2. Characterization of the semiconducting film's stretchability and electrical performance under different strains. (A) Schematic illustration of the stretching of the semiconducting film supported on PDMS substrate. (B) Optical microscope images of a neat-1 film (left, top) and a CONPHINE-1 film (left, bottom) under 100\% strain, with AFM phase image showing the detailed morphology of the CONPHINE-1 film. (C) Schematic of the applied soft contact lamination method to derive the electrical performance of a semiconducting layer upon stretching. (D) Transfer curves $\left(\mathrm{V}_{\mathrm{D}}=-80 \mathrm{~V}\right)$ obtained from the CONPHINE-1 film and the neat-1 film in its original condition (i), under 100\% strain parallel to the charge transport direction (ii), and under $100 \%$ strain perpendicular to the charge transport direction (iii). (E, F) Mobilities from the CONPHINE-1 film (blue) and the neat-1 film (black) at different strains (E) parallel and (F) perpendicular to the charge transport direction. (G) Comparison of the obtained mobilities at stretched strains in this paper to the previously reported results in the references. (H) Mobilities of the CONPHINE-1 film (green) and the neat-1 film (black) as a function of 100\%-strain stretching cycles.

To evaluate its mechanical robustness, effect of multiple stretch-release cycles was investigated. First, for the CONPHINE-1 film, the on-current only changes 
moderately after 100 cycles with $100 \%$ strain (Fig. S15E), and the mobility is still high at $0.3 \mathrm{~cm}^{2} /$ Vs (Fig. $2 \mathrm{H}$ ). Furthermore, there are no visible cracks in the film after 100 cycles (Fig. S18); however, we do observe that there is slightly increase in film roughness (Fig. S19), which might lead to the slight decrease in mobility as a result of the affected contact to the electrodes. In comparison, for the neat-1 film, both the on-current and mobility greatly decreased by $>3$ orders after the 100 cycles (Figs. $2 \mathrm{H}$ and $\mathrm{S} 15 \mathrm{~F}$ ). Compared to the reported cyclability at strains lower than $50 \%$ in literatures $(6,15,27)$, the mobility of our CONPHINE film after cycled stretching is over 1 order higher.

With our high-stretchability and high-mobility CONPHINE semiconducting film, we further fabricate bottom-gate-bottom-contact fully stretchable transistors, with carbon nanotube (CNT) network as the electrodes, SEBS as the dielectric layer, the stretchable substrate and also the encapsulation layer on the top (Figs. 3A \& S20). As displayed in Fig. 3B, the obtained TFT device has good transparency and excellent conformability for constructing e-skins on human epidermis. As shown via a representative transfer curve (Fig. 3C) and output characteristics (Fig. S21A), the fully stretchable TFT gives ideal transistor performance, with an average mobility of $0.59 \mathrm{~cm}^{2} / \mathrm{Vs}$. The fabricated arrays of TFTs also have high uniformity in performance, with minimal variations in mobility among 20 devices (Fig. 3D). The lower mobility compared to that obtained from the earlier soft contact lamination method is attributed to a higher contact resistance from CNT electrode than Au (Fig. S22). We note that besides having outstanding stretchability, the CONPHINE-1 semiconductor layer 
(containing $70 \mathrm{wt} \%$ SEBS content) also has excellent adhesion to both the SEBS dielectric and encapsulation layer. When the device is stretched to $100 \%$ strain along the charge transport direction, the transfer curve remains unaffected (Fig. S21B), albeit with only a very slight drop in the on-current due to the increase of channel length from stretching. In addition, there is almost no decrease in the mobility, with a value of $0.55 \mathrm{~cm}^{2} / \mathrm{Vs}$ at $100 \%$ strain (Fig. 3E), which is again a record-high value compared to previously reported fully stretchable organic transistors (Table S2). Along the perpendicular direction, the conjunctional effect of the slight decrease in mobility and the device geometric change makes the transfer curve highly stable under strain up to $100 \%$ (Figs. 3E \& S21C). Moreover, this fully stretchable TFT is highly robust over 1000 repeated stretching cycles, as shown in Figs. 3F \& S21D. The excellent stretchablity and robustness of the TFT is also clearly revealed by its highly stable drain-current under sequential stretching, twisting and poking by a sharp object, as shown in Fig. 3G and Movie S1.

Due to its outstanding stretchability and transparency, our fully stretchable TFT made from CONPHINE-1 semiconductor is promising for wearable electronics. To demonstrate its utility, we proceed to conformably attach our transistor, serving as a LED driver, to a human finger. During the repeated bending of the finger that created a large strain $(>50 \%)$ on the device, the intensity of the light from the LED remains unaffected (Fig. 3H \& Movie S2), indicating its robustness and, more importantly, its suitability as a wearable display system and other stretchable electronic systems. 


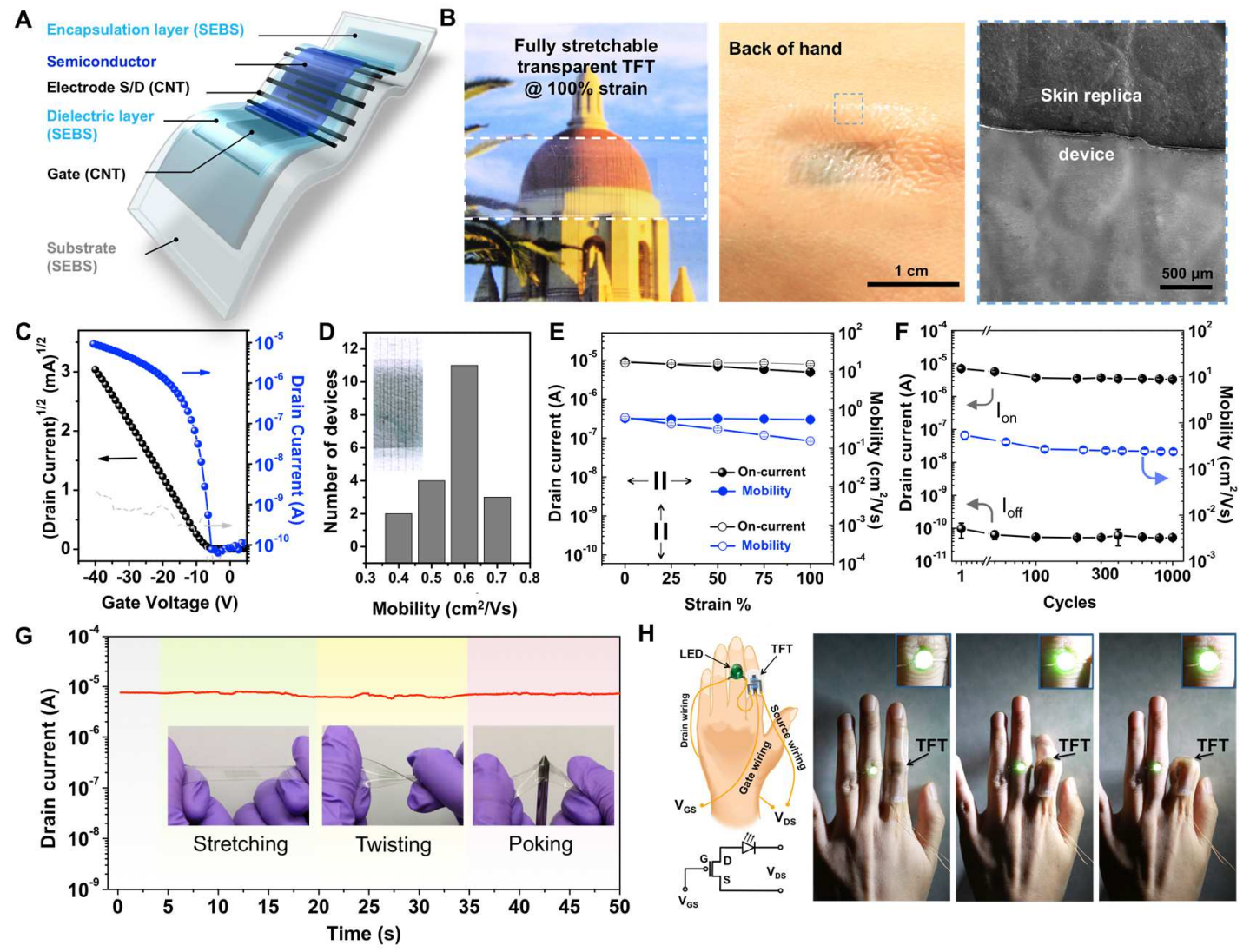

Fig. 3. Fully stretchable transistor device fabricated from the CONPHINE-1 film. (A)

Structure of the fully stretchable transistor. (B) Pictures showing the transparency of the fully stretchable TFT (left), the skin-like nature of the device attached on the back of hand (middle), with the conformability shown in the SEM image (right). (C) A typical transfer curve $\left(V_{D}=\right.$ $-30 \mathrm{~V}$ ) from the fully stretchable transistor at $0 \%$ strain. (D) Distribution of the mobility from 20 devices in the arrays of fully stretchable transistors (showing with increased contrast in the inset). (E) Changes of the on-current and the mobility with strains up to $100 \%$, both parallel to (solid points) and perpendicular to (hollow points) the charge transport direction (F) Changes of the on-current, the off-current and the mobility with strain along the charge transport direction, and (D), and perpendicular to the charge transport direction (F). Changes of the on-current, the off-current and the mobility after multiple stretching-releasing cycles (up to 1000 cycles) at $25 \%$ strain along the charge transport direction. (G) Drain-current of a fully stretchable TFT under sequential stretching, twisting and poking on a shape object. (H) Demonstration of the fully stretchable transistor attached as the electronic skin of a bending finger while driving an LED. 
For broad applicability, our concept demonstrated here in using nanoconfinement effect to significantly improve the stretchability of polymer semiconductor films should serve to benefit various other conjugated polymers. Hence, we next apply our developed CONPHINE method to four other high-performance semiconducting polymers (2-5), with their chemical structures shown in Figs. 4A-D (36-38). First, all the neat films of these polymers were observed to be severely cracked when subjected to $100 \%$ strain (middle column images in Figs. 4A-D). Thus, their measured mobilities are all detrimentally degraded as marked in their respective images. On the other hand, upon using our CONPHINE method, nanoconfined morphologies with deformable interfaces are again obtained. (Fig. S23). The stretchability of all these polymers (CONPHINE-2 to CONPHINE-5) is significantly improved, although we do observe inhomogeneous deformations at 100\% strain (right column images in Figs. 4A-D \& S24). As a result, both the on-currents and mobilities (values are indicated in their respective images) from these CONPHINE-2 to CONPHINE-5 films at $100 \%$ strain are all much higher than their neat counterparts by one to four orders of magnitude (Figs. 4E \& F). Hence, through our CONPHINE method, four different conjugated polymers (including 1) have been demonstrated with mobilities $>1.0$ $\mathrm{cm}^{2} / \mathrm{Vs}$ at $100 \%$ strain. 
A

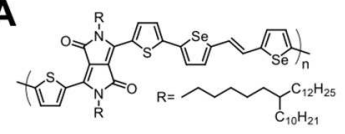

P-29-DPPDTSE (2)

B

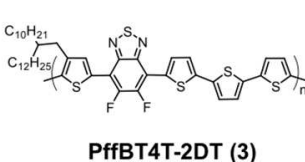

C

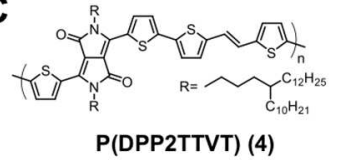

D

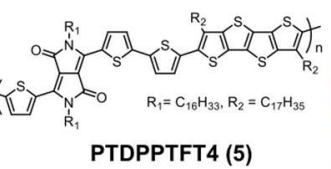

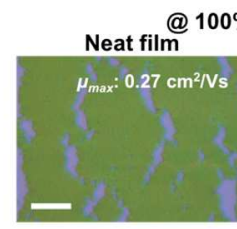
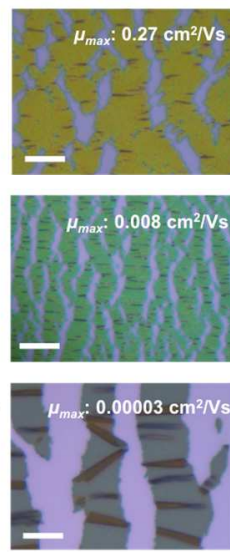
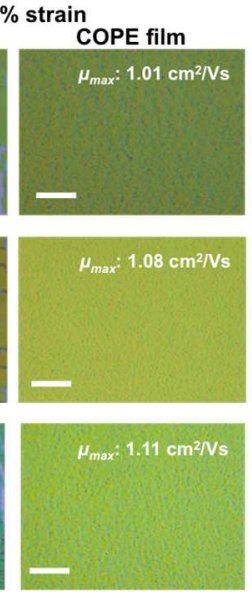

$\mu_{\max }: 0.21 \mathrm{~cm}^{2} \mathrm{Ns}$
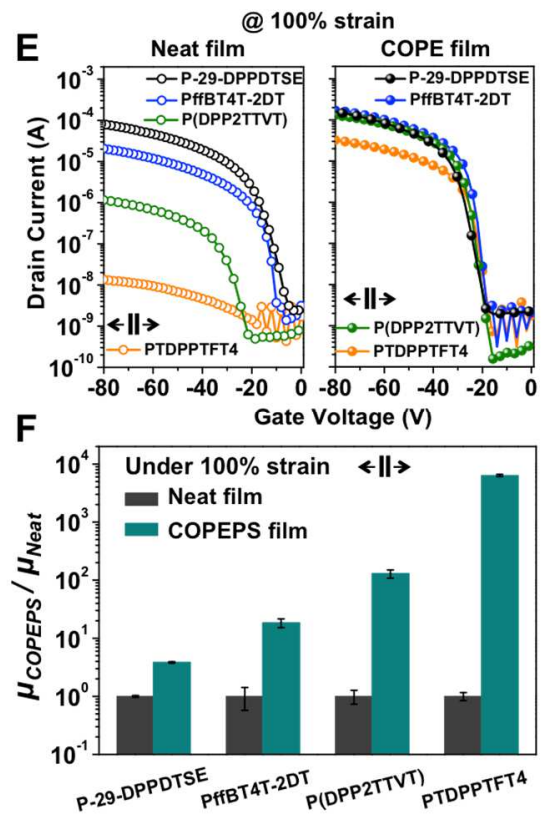

Fig. 4. Applying the CONPHINE method on four distinct conjugated semiconducting polymers to improve their stretchability. (A-D) Optical micrographs of neat conjugated polymer films (left) and corresponding CONPHINE films (right) at 100\% strain, for polymers of (A) P-29-DPPDTSE; 2, (B) PffBT4T-2DT; 3, (C) P(DPP2TTVT); 4, and (D) PTDPPTFT4; 5, with the chemical structures shown on the right side. Scale bar $=20 \mu \mathrm{m}$. The CONPHINE films at $100 \%$ strain exhibited un-even thickness (Fig. S23), but no cracks due to plastic deformation. (E) Transfer curves $\left(V_{D}=-80 \mathrm{~V}\right)$ of these neat polymer films (in the left plot) and corresponding CONPHINE films (in the right plot) at 100\% strain, with the same vertical axis in the two diagrams. (F) Normalized mobilities of neat films (grey) and corresponding CONPHINE films (green) of these conjugated polymers, under 100\% strain.

In summary, the concept of nanoconfinement effect is utilized to enable high stretchablity in polymer semiconducting materials. In specific, we introduce a conjugated-polymer-elastomer phase-separation induced engineering (CONPHINE) method to create conjugated-polymer nanostructures with increased chain dynamics embedded in an elastomer matrix to afford significant increases in various semiconducting film's mechanical ductility while maintaining their performance 
parameters. This new concept provides several advantages, including significant improvement to the polymer semiconductor mechanical stretchability in both directions to the charge transport, no reduction in the semiconductor charge transport performance, simplicity in fabrication, wide applicability to different semiconducting polymers, and enhanced cost efficiency due to the reduced usage of conjugated polymers in semiconducting layer. Besides the CONPHINE method described, nanoconfinement effect may also be realized by other patterning/templating methods. We anticipate this general approach to significantly advance the development of stretchable semiconductors for stretchable electronic-skin applications.

\section{REFERENCES AND NOTES}

1. D.-H. Kim et al., Epidermal electronics. science 333, 838-843 (2011).

2. D.-H. Kim et al., Materials for multifunctional balloon catheters with capabilities in cardiac electrophysiological mapping and ablation therapy. Nature materials 10, 316-323 (2011).

3. B. C. K. Tee et al., A skin-inspired organic digital mechanoreceptor. Science 350, 313-316 (2015).

4. S. Lee et al., A strain-absorbing design for tissue-machine interfaces using a tunable adhesive gel. Nature communications 5, (2014).

5. Y. Sun, W. M. Choi, H. Jiang, Y. Y. Huang, J. A. Rogers, Controlled buckling of semiconductor nanoribbons for stretchable electronics. Nature nanotechnology 1, 201-207 (2006).

6. A. Chortos et al., Highly Stretchable Transistors Using a Microcracked Organic Semiconductor. Advanced Materials 26, 4253-4259 (2014).

7. S. P. Lacour, D. Chan, S. Wagner, T. Li, Z. Suo, Mechanisms of reversible stretchability of thin metal films on elastomeric substrates. Applied Physics Letters 88, 204103 (2006). 
8. T. C. Shyu et al., A kirigami approach to engineering elasticity in nanocomposites through patterned defects. Nature materials, (2015).

9. C. B. Nielsen, M. Turbiez, I. McCulloch, Recent Advances in the Development of Semiconducting DPP-Containing Polymers for Transistor Applications. Advanced Materials 25, 1859-1880 (2013).

10.H.-R. Tseng et al., High mobility field effect transistors based on macroscopically oriented regioregular copolymers. Nano letters 12, 6353-6357 (2012).

11.H. N. Tsao et al., Ultrahigh mobility in polymer field-effect transistors by design. Journal of the American Chemical Society 133, 2605-2612 (2011).

12.S. Savagatrup, A. D. Printz, D. Rodriquez, D. J. Lipomi, Best of Both Worlds: Conjugated Polymers Exhibiting Good Photovoltaic Behavior and High Tensile Elasticity. Macromolecules 47, 1981-1992 (2014).

13.S. Savagatrup, A. S. Makaram, D. J. Burke, D. J. Lipomi, Mechanical Properties of Conjugated Polymers and Polymer-Fullerene Composites as a Function of Molecular Structure. Advanced Functional Materials 24, 1169-1181 (2014).

14.E. Song et al., Stretchable and Transparent Organic Semiconducting Thin Film with Conjugated Polymer Nanowires Embedded in an Elastomeric Matrix. Advanced Electronic Materials 2, (2016).

15.J. Y. O. Minkwan Shin, Kyung-Eun Byun , Yu-Jeong Lee, Bongsoo Kim „, J.-J. P. Hong-Koo Baik , and Unyong Jeong, Polythiophene Nanofi bril Bundles Surface-Embedded in Elastomer: A Route to a Highly Stretchable Active Channel Layer. Advanced Materials, (2015).

16.C. M. Stafford, B. D. Vogt, C. Harrison, D. Julthongpiput, R. Huang, Elastic moduli of ultrathin amorphous polymer films. Macromolecules 39, 5095-5099 (2006).

17.J. Martin, M. Munoz, M. Encinar, M. Calleja, M. Martin-Gonzalez, Fabrication and Mechanical Characterization of Semi-Free-Standing (Conjugated) Polymer Thin Films. Langmuir 30, 5217-5223 (2014).

18.J. L. Keddie, R. A. L. Jones, R. A. Cory, SIZE-DEPENDENT DEPRESSION OF THE GLASS-TRANSITION TEMPERATURE IN POLYMER-FILMS. Europhysics Letters 27, 59-64 (1994). 
19.C. J. Ellison, J. M. Torkelson, The distribution of glass-transition temperatures in nanoscopically confined glass formers. Nature Materials 2, 695-700 (2003).

20.L. Si, M. V. Massa, K. Dalnoki-Veress, H. R. Brown, R. A. L. Jones, Chain entanglement in thin freestanding polymer films. Physical Review Letters 94, (2005).

21.B. J. Gurmessa, A. B. Croll, Onset of Plasticity in Thin Polystyrene Films. Physical Review Letters 110, (2013).

22.J.-H. Lee, J. Y. Chung, C. M. Stafford, Effect of Confinement on Stiffness and Fracture of Thin Amorphous Polymer Films. Acs Macro Letters 1, 122-126 (2012).

23.P. Ray, K. Binder, Finite-size effect in the dynamics near the glass transition. EPL (Europhysics Letters) 27, 53 (1994).

24.C. L. Soles, Y. Ding, MATERIALS SCIENCE Nanoscale Polymer Processing. Science 322, 689-690 (2008).

25.J. A. Forrest, K. DalnokiVeress, J. R. Dutcher, Interface and chain confinement effects on the glass transition temperature of thin polymer films. Phys. Rev. E 56, 5705-5716 (1997).

26.K. Shin et al., Crystalline structures, melting, and crystallization of linear polyethylene in cylindrical nanopores. Macromolecules 40, 6617-6623 (2007).

27.H.-C. Wu et al., A Rapid and Facile Soft Contact Lamination Method: Evaluation of Polymer Semiconductors for Stretchable Transistors. Chemistry of Materials 26, 4544-4551 (2014).

28.R. Peng et al., An ABA triblock copolymer strategy for intrinsically stretchable semiconductors. Journal of Materials Chemistry C 3, 3599-3606 (2015).

29.B. O'Connor et al., Anisotropic Structure and Charge Transport in Highly Strain-Aligned Regioregular Poly(3-hexylthiophene). Advanced Functional Materials 21, 3697-3705 (2011).

30.M. Shin et al., Highly stretchable polymer transistors consisting entirely of stretchable device components. Advanced Materials 26, 3706-3711 (2014).

31.Y. Li, S. P. Singh, P. Sonar, A High Mobility P-Type DPP-Thieno [3, 2-b] thiophene Copolymer for Organic Thin-Film Transistors. Advanced Materials 22, 4862-4866 (2010).

32.J. F. Chang, M. C. Gwinner, M. Caironi, T. Sakanoue, H. Sirringhaus, ConjugatedPolymer-Based Lateral Heterostructures Defined by High-Resolution Photolithography. 
Advanced Functional Materials 20, 2825-2832 (2010).

33.J. Kim et al., Density of nanopatterned surfaces for designing bone tissue engineering scaffolds. Materials Letters 130, 227-231 (2014).

34.S. Wang et al., Experimental evidence that short-range intermolecular aggregation is sufficient for efficient charge transport in conjugated polymers. Proceedings of the National Academy of Sciences of the United States of America 112, 10599-10604 (2015).

35.Z. Suo, J. Vlassak, S. Wagner, Micromechanics of macroelectronics. China Particuology 3, 321-328 (2005).

36.I. Kang, H.-J. Yun, D. S. Chung, S.-K. Kwon, Y.-H. Kim, Record High Hole Mobility in Polymer Semiconductors via Side-Chain Engineering. Journal of the American Chemical Society 135, 14896-14899 (2013).

37.Y. Liu et al., Aggregation and morphology control enables multiple cases of high-efficiency polymer solar cells. Nature communications 5, (2014).

38.W. Y. Lee et al., Effect of Non-Chlorinated Mixed Solvents on Charge Transport and Morphology of Solution-Processed Polymer Field-Effect Transistors. Advanced Functional Materials 24, 3524-3534 (2014). 